\title{
Construction of a Choquet integral and the value functions without any commensurateness assumption in multi-criteria decision making
}

\author{
Christophe Labreuche
}

Thales Research \& Technology, 1 avenue Augustin Fresnel, 91767 Palaiseau Cedex - France

\begin{abstract}
We consider a multi-criteria evaluation function $U$ defined over a Cartesian product of attributes. We assume that $U$ is written as the combination of an aggregation function and one value function over each attribute. The aggregation function is assumed to be a Choquet integral w.r.t. an unknown capacity. The problem we wish to address in this paper is the following one: if $U$ is known, can we construct both the value functions and the capacity? The approaches that have been developed so far in the literature to answer this question in an analytical way assume some commensurateness hypothesis. We propose in this paper a method to construct the value functions and the capacity without any commensurateness assumption. Moreover, we show that the construction of the value functions is unique up to an affine transformation.
\end{abstract}

Keywords: Choquet integral, capacity, value functions, commensurateness.

\section{Introduction}

One of the most widely used model in decision theory is the weighted sum. The overall value is then written as a weighted sum of value functions on each attribute. This model has been extensively studied in the context of decision under uncertainty $[1,2]$ and multi-criteria decision making [3]. The main drawback of this model is that it assumes the independence among the criteria. The Choquet integral which subsumes the weighted sum, has been used in decision theory to overcome the limitation of the weighted sum [4]. The Choquet integral is a versatile aggregation function that has been applied to Multi-Criteria Decision Analysis [5] and Data fusion. Its main interest lies in its ability to represent interaction among the attributes. The overall value for this model is written as the Choquet integral of value functions on each attribute.

Unlike the weighted sum, the use of the Choquet integral implies that the scales representing the attributes are commensurate. So far, there is no work in which both the Choquet integral and the value functions are constructed in an analytical way with- out making any a priori assumption about commensurateness.

The determination of not only the admissible capacities but also the utility functions has been considered from a practical side. The existing methods to solve this problem as a whole are based on stochastic approaches (Monte Carlo or genetic algorithm), as it is the case in [6]. In [7], the value functions and the Choquet integral are constructed under the assumption that one already knows two reference elements on each attribute that are commensurate. From a measurement standpoint, an interval scale is uniquely specified up to two degrees of freedom, and it is sufficient to fix the scale on two reference elements to completely fix the scale. In [8], the Choquet integral is axiomatized as an aggregation function. There is no value function in this approach. In [4], the preference relation provided by the Choquet integral is axiomatized in the context of decision under uncertainty. In this setting, there is no commensurateness issue since all attributes and thus all value functions are identical. In [9], the Choquet integral is axiomatized as an aggregation function in the context of decision under uncertainty. In [10], the neutral element of a bipolar scale is determined under the knowledge that the decision maker satisfies a special decision strategy called conditional relative importance between criteria. In this work, by analyzing the non linear decision strategies represented in the overall utility function, it is possible to identify the neutral value in the value functions. This idea will be used and extended in this paper.

In the world of fuzzy integrals, let us mention that our objective in this paper is already reached for the Sugeno integral. In $[11,12,13,14]$, the preference relation of the Sugeno integral is axiomatized with different and unknown value functions. It is easier to recover the value functions through a Sugeno integral than for the Choquet integral since the Sugeno integral behaves as a median. The idea is that, if the value on one attribute varies (and the values on the other attributes being fixed), then the value function on this attribute is equal to the overall utility when this latter is not constant.

The main idea of this paper is now summarized. Commensurateness between the criteria is required for the Choquet integral since this aggregation func- 
tion is based a ranking of the values of the criteria. Yet, the Choquet integral is a piecewise weighted sum function, in which the weights of the criteria are the same whenever the criteria are ranked in the same order. Considering two criteria $i$ and $k$, the weight of criterion $i$ depends on the relative values of criteria $i$ and $k$. This means that, if the value of criterion $k$ varies and the other criteria are fixed, then one may observe that the weight of criterion $i$ suddenly changes when the value of criterion $k$ is equal to that of criterion $i$. From this remark, it is possible to construct, from an element of the attribute $i$, an element of the attribute $k$ that is commensurate to the previous element. This construction does not work if the weight of criterion $i$ does not depend on criterion $k$. If this holds for any value on the other criteria, one can show that this implies that the criteria $i$ and $k$ are independent. Applying this construction to any pair $i, k$ of criteria, one obtains a partition of the set of criteria. In each set, the criteria interact one with another, and it is thus possible to construct vectors of values on the attributes that are commensurate. There is complete independence between the criteria of any two sets in this partition. Hence one cannot ensure commensurateness between two sets in the partition. But this is not a problem since the Choquet integral is additive between groups of criteria that are independent.

The layout of the paper is as follows. Section 3 recalls some useful results on the construction of value functions. Our major difficulty in the construction of the value function is to construct two reference values on each attribute that are commensurate. This is done in Section 4, based on the construction that we have just presented. When two sequences of commensurate values are obtained, we are able to construct the value functions and the capacity by generalizing the approach of [7] to the case when the commensurateness is attained only within each set in a partition of the criteria (see Section 5).

\section{Notation and the Choquet integral}

\subsection{Preference relation}

Consider a problem of selecting one option among several, where each option is described by several attributes. $N=\{1, \ldots, n\}$ is the set of attributes and the set of possible values of attribute $i \in N$ is denoted by $X_{i}$. Options are thus elements of the product set $X:=X_{1} \times \cdots \times X_{n}$. The preferences of the DM over the options can be described by a preference relation $\succsim$ over $X$. For $x, y \in X, x \succsim y$ means that $x$ is at least as good as $y$ according to the DM.

Considering two acts $x, y \in X$ and $S \subseteq N$, we use the notation $\left(x_{S}, y_{-S}\right)$ to denote the compound act $w \in X$ such that $w_{i}=x_{i}$ if $i \in S$ and $y_{i}$ otherwise. Likewise, options $\left(x_{S}, y_{T}, z_{-S \cup T}\right)$ denotes the compound act $w \in X$ such that $w_{i}=x_{i}$ if $i \in S$, $w_{i}=y_{i}$ if $i \in T$, and $w_{i}=z_{i}$ otherwise.

Representing $\succsim$ by a numerical scale demands to address two issues: the preferences over each attribute and the aggregation of these preferences. Speaking of a preference relation focusing only on one attribute implies that the other attributes could have been removed. The existence of a preference relation on each attribute classically relies on weak separability [3].

Definition 1 A relation $\succsim$ is said to be weakly separable if for every $i \in N$, and for all $x_{i}, x_{i}^{\prime} \in X_{i}$, $y_{N \backslash\{i\}}, y_{N \backslash\{i\}}^{\prime} \in X_{N \backslash\{i\}}$,

$$
\begin{aligned}
&\left(x_{i}, y_{-i}\right) \succsim\left(x_{i}^{\prime}, y_{-i}\right) \\
& \Longleftrightarrow\left(x_{i}, y_{-i}^{\prime}\right) \succsim\left(x_{i}^{\prime}, y_{-i}^{\prime}\right) .
\end{aligned}
$$

Then, for $i \in N$, the marginal preference relation $\succsim_{i}$ on attribute $i$ is defined on $X_{i}$ as follows

$$
\begin{aligned}
x_{i} \succsim_{i} y_{i} & \Longleftrightarrow \\
\forall z_{N \backslash\{i\}} & \in X_{N \backslash\{i\}},\left(x_{i}, z_{-i}\right) \succsim\left(y_{i}, z_{-i}\right) .
\end{aligned}
$$

This property can be interpreted as a weak independence between attributes. This assumption is essential for quantitative models based on an overall value function. From [3], $\succsim$ can be represented by functions $u_{i}: X_{i} \rightarrow \mathbb{R}$ and $F: \mathbb{R}^{n} \rightarrow \mathbb{R}:$

$$
x \succsim y \quad \Longleftrightarrow \quad U(x) \geq U(y)
$$

where

$$
U(x)=F\left(u_{1}\left(x_{1}\right), \ldots, u_{n}\left(x_{n}\right)\right)
$$

iff $\succsim$ is a weak order, is weakly separable and satisfies a technical topological assumption.

\subsection{Choquet integral}

A game on a set $N$ of criteria is a set function $\mu$ : $2^{N} \rightarrow[0,1]$ such that $[15]$

- $\mu(\emptyset)=0$

- $\forall A \subseteq B \subseteq N, \mu(A) \leq \mu(B)$

A capacity (also called fuzzy measure) on a set $N$ of criteria is a game for which $\mu(N)=1$ [16]. This condition is a normalization condition. Hence games are called non-normalized capacities.

In the context of multi-criteria decision making, the normalization condition is important since it is related to the idempotency of the aggregation function.

The Choquet integral of $x=\left(x_{1}, \ldots, x_{n}\right) \in[0,1]^{n}$ defined w.r.t. a game $\mu$ has the following expression 
[17] :

$$
\begin{aligned}
& C_{\mu}\left(a_{1}, \ldots, a_{n}\right) \\
& =\sum_{i=1}^{n}\left(a_{\tau(i)}-a_{\tau(i-1)}\right) \mu(\{\tau(i), \cdots, \tau(n)\}),
\end{aligned}
$$

where $a_{\tau(0)}:=0$ and $a_{\tau(1)} \leq a_{\tau(2)} \leq \cdots \leq a_{\tau(n)}$. The Choquet integral has been proved to be able to model both the importance of criteria and the interaction between criteria.

The Möbius transform of $\mu$, denoted by $m$, is given by

$$
m(A)=\sum_{B \subseteq A}(-1)^{|A \backslash B|} \mu(B) .
$$

The expression of the Choquet integral w.r.t. the Möbius transform is

$$
C_{\mu}\left(a_{1}, \ldots, a_{n}\right)=\sum_{A \subseteq N} m(A) \bigwedge_{i \in A} a_{i} .
$$

\subsection{Interpretation}

A capacity is relatively complex to interpret since it contains $2^{n}$ coefficients. It can be easily interpreted in term of the importance of criteria and the interaction between criteria.

For $i \in N$ and $A \subseteq N \backslash\{i\}$, we define the discrete derivative $\delta_{i}$ by:

$$
\delta_{i} \mu(A)=\mu(A \cup\{i\})-\mu(A) .
$$

For $i, j \in N$ with $i \neq j$, and $A \subseteq N \backslash\{i, j\}$, we define the discrete derivative of order $2 \delta_{i, j}$ by:

$\delta_{i, j} \mu(A):=\mu(A \cup\{i, j\})-\mu(A \cup\{i\})-\mu(A \cup\{j\})+\mu(A)$.

Let $\mu$ be a game on $N$. The Shapley index [18] for every $i \in N$ is defined by

$$
v_{i}:=\sum_{A \subset N \backslash\{i\}} \frac{|A| !(n-|A|-1) !}{n !} \delta_{i} \mu(A)
$$

The Shapley index $v_{i}$ can be interpreted as a kind of average value of the contribution of element $i$ alone in all coalitions. A basic property of the Shapley value is that $\sum_{i=1}^{n} v_{i}=\mu(N)$.

For a game $\mu$ on $N$, we define interaction index [19] of criteria $i, j$ by

$$
I_{i j}:=\sum_{A \subset N \backslash\{i, j\}} \frac{|A| !(n-|A|-2) !}{(n-1) !} \delta_{i, j} \mu(A) .
$$

The interaction index $I_{i j}$ can be interpreted as a kind of average value of the added value given by putting $i$ and $j$ together, all coalitions being considered. It is positive when $i, j$ act in a cooperative or complementary way (i.e. the importance of the pair $(i, j)$ is significantly greater than importances of $i$ and $j$ alone), and it is negative when $i, j$ act in a redundant or substitutive way (i.e. the importance of the pair $(i, j)$ is almost the same as importances of $i$ and $j$ alone). It is zero if the criteria $i$ and $j$ are independent. It is the case with a weighted sum: $I_{i j}=0$ for any pair $i, j$ of criteria.

\section{Related works on the construction of value functions}

\subsection{Aggregation model that allows simple construction of the value functions}

There is in [20] (see also [21]) a major result regarding the construction of value functions in conjoint measurement. The aim is to construct a measurable value function $v: X \rightarrow \mathbb{R}$ from preference order relations. Classically, this is done through a binary relation $\succsim$ over $X$ (where $x \succsim y$ means that $x \in X$ is at least as good as $y \in X$ ) and a quaternary relation $\succsim^{\star}$ over $X$ (where $x y \succsim^{\star} z t$ means that the strength of preference for $x \in X$ over $y \in X$ is greater or equal to the strength of preference for $z \in X$ over $t \in X)$. A function $v$ satisfying to the two natural properties

$$
\begin{aligned}
x \succsim y & \Longleftrightarrow v(x) \geq v(y), \\
x y \succsim^{\star} z t & \Longleftrightarrow v(x)-v(y) \geq v(z)-v(t) .
\end{aligned}
$$

is called a measurable value function.

It is well-known that an interval scale can be constructed from a knowledge of a binary relation and a quaternary relation written in terms of difference of values [3]. In the MACBETH approach, the quaternary relation $\succsim^{\star}$ is constructed from categories of intensity of preferences [22]. The AHP (Analytical Hierarchy Process) [23], is not based on a quaternary relation but on ratios. The decision maker is asked to assess the ratio between the values, and is thus based on the relation $\overbrace{}^{\diamond}$ defined by

$$
x y \succsim^{\diamond} z t \quad \Longleftrightarrow \quad \frac{v(x)}{v(y)} \geq \frac{v(z)}{v(t)} .
$$

This implies that $v$ is a ratio scale, which is very seldom the case. Moreover, individuals have many difficulties in assessing ratios. Henceforth, we will consider an interval scale defined by $\succsim$ and $\succsim^{\star}$.

Attribute $i$ is said to be weak difference independent if given any $x_{i}, y_{i}, z_{i}, w_{i} \in X_{i}$ and any $t_{-i} \in$ $X_{-i}$ such that $\left(x_{i}, t_{-i}\right)\left(y_{i}, t_{-i}\right) \succsim^{\star}\left(z_{i}, t_{-i}\right)\left(w_{i}, t_{-i}\right)$, then $\left(x_{i}, s_{-i}\right)\left(y_{i}, s_{-i}\right) \succsim^{\star}\left(z_{i}, s_{-i}\right)\left(w_{i}, s_{-i}\right)$ for any $s_{-i} \in X_{-i}[20]$. This property is the counterpart of weakly separability applied to $\succsim^{\star}$.

This property allows to construct the value function on attribute $i$ by asking questions directly regarding the preference of the DM on the set $X_{i}$ (independently on the values on the other attributes) rather than from questions regarding options in $X$ (e.g. from a subset of $\succsim$ and $\succsim^{\star}$ ).

Proposition 1 (Corollary 2 in [20] applied to Theorem 6.3 in [21]) Assume that $n \geq 2$. Then $v$ is a measurable value function that fulfills weak difference independent for each attribute if and only if $v$ is the multi-linear value model $v(x)=M_{\mu}\left(u_{1}\left(x_{1}\right), \ldots, u_{n}\left(x_{n}\right)\right)$ w.r.t. a capacity $\mu$, where $M_{\mu}\left(a_{1}, \ldots, a_{n}\right)=\sum_{A \subseteq N} m(A) \prod_{i \in A} a_{i}$ (where $m$ is the Möbius transform of $m$ ). 
Note that the multi-linear value model $M_{\mu}$ is close to the expression of the Choquet integral in terms of the Möbius transform (see (4)).

From this result, the Choquet integral does not fulfill the weak difference independent property. Hence the construction of the value functions through the Choquet integral is not an easy task.

\subsection{An existing approach to construct the value functions with the Choquet integral}

The reason why the Choquet integral fails to satisfy the weak difference independent property is that the criteria are ranked in the expression of the Choquet integral and need thus to be commensurate. In order to ensure commensurateness among the criteria, the approach of $[7,24]$ is to extend the MACBETH methodology (see [22]). The main idea is that one needs to identify two reference values (denoted by (1) and $\mathbb{G}$ - where (1) stands for an unacceptable value, and $\mathbb{G}$ stands for Good) on each scale to completely fix the value functions. On each attribute set $X_{i}$, it is assumed that one can identify an element $\mathbb{O}_{i} \in X_{i}$ that is judged unacceptable, and an element $\mathbb{G}_{i} \in X_{i}$ that is judged perfectly satisfactory. All reference elements $\mathbb{O}_{1}, \mathbb{O}_{2}, \ldots, \mathbb{O}_{n}$ (resp. $\left.\mathbb{G}_{1}, \mathbb{G}_{2}, \ldots, \mathbb{G}_{n}\right)$ are commensurate and should be assigned the same value - e.g. 0 (resp. 1). The difficulty of the construction of the value functions is that the capacity $\mu$ is not known, and one needs to construct them through the aggregation function. The construction of the value function on $X_{i}$ is performed by only asking information regarding elements of $\left.X_{i}\right\rfloor:=\left\{\left(x_{i}, \mathbb{O}_{-i}\right): x_{i} \in X_{i}\right\}[7]$. Let $V_{i}$ be any scale representing the preference of the decision maker over $\left.X_{i}\right\rfloor$. Then the value function $u_{i}$ is obtained from $V_{i}$ by a dilation and shift: $u_{i}\left(x_{i}\right)=\frac{V_{i}\left(x_{i}, \mathbb{O}_{-i}\right)-V_{i}\left(\mathbb{O}_{N}\right)}{V_{i}\left(\mathbb{G}_{i}, \mathbb{O}_{-i}\right)-V_{i}\left(\mathbb{O}_{N}\right)}$ for all $x_{i} \in X_{i}$. From this construction, the right normalization of $u_{i}$ is obtained: $u_{i}\left(\mathbb{O}_{i}\right)=0$ and $u_{i}\left(\mathbb{G}_{i}\right)=1$.

\section{Construction of profiles of commensurate values}

\subsection{Basic assumptions}

From now on, we assume that we know the overall value $U(x)$ for all $x \in X$. It is supposed to take the form for all $x \in X$,

$$
U(x)=C_{\mu}\left(u_{1}\left(x_{1}\right), \ldots, u_{n}\left(x_{n}\right)\right)
$$

The aim is to construct $\mu$ and $u_{1}, \ldots, u_{n}$.

For the sake of simplicity, we assume that

$$
X=\mathbb{R}^{n},
$$

and that the value functions are strictly increasing. This can be expressed in terms of the overall value function $U$ as follows:

$$
\begin{array}{r}
\forall x_{i}, y_{i} \in X_{i} \text { with } x_{i}>y_{i} \quad \exists z_{-i} \in X_{-i} \\
\text { such that } U\left(x_{i}, z_{-i}\right)>U\left(y_{i}, z_{-i}\right) .
\end{array}
$$

It is easy to show that the previous relation is indeed equivalent to the fact that $u_{i}$ is strictly increasing, provided that the capacity $\mu$ does not rule out one criterion.

Furthermore, we assume that $U$ is continuous and that for all $i \in N$, there exists $x_{-i} \in X_{-i}$ such that

$$
\lim _{x_{i} \rightarrow+\infty} U(x)=+\infty \text { and } \lim _{x_{i} \rightarrow-\infty} U(x)=-\infty .
$$

\subsection{Commensurateness between two criteria}

Let us consider in this section, two criteria $i, k \in$ $N$ with $i \neq k$. For $\varepsilon>0$ and $x_{i} \in X_{i}$, we set $x_{i}^{\varepsilon}:=x_{i}+\varepsilon$. As depicted in the introduction, we fix the values $x_{-k}$ on all attributes except attribute $k$, and we analyze the influence of $x_{k}$ on the difference $U\left(x_{i}^{\varepsilon}, x_{-i}\right)-U(x)$. This difference is proportional to the weight of criterion $i$. Lemma 1 below gives the expression of this difference for all values of $x_{k}$, for $\varepsilon>0$ small enough.

For $x \in X$, we set

$$
A_{>i}(x)=\left\{j \in N: u_{j}\left(x_{j}\right)>u_{i}\left(x_{i}\right)\right\} .
$$

Lemma 1 Let $x \in X$. Let $\varepsilon>0$ sufficiently small so that $\left(u_{j}\left(x_{j}\right)\right)_{j \in N \backslash\{k\}}$ and $\left(u_{i}\left(x_{i}^{\varepsilon}\right),\left(u_{j}\left(x_{j}\right)\right)_{j \in N \backslash\{i, k\}}\right)$ are comonotone (without considering criterion $k$ ).

Then if $u_{k}\left(x_{k}\right) \leq u_{i}\left(x_{i}\right)$

$$
U\left(x_{i}^{\varepsilon}, x_{-i}\right)-U(x)=\left(u_{i}\left(x_{i}^{\varepsilon}\right)-u_{i}\left(x_{i}\right)\right) \delta_{i} \mu(S),
$$

if $u_{i}\left(x_{i}\right) \leq u_{k}\left(x_{k}\right) \leq u_{i}\left(x_{i}^{\varepsilon}\right)$, then

$$
\begin{aligned}
& U\left(x_{i}^{\varepsilon}, x_{-i}\right)-U(x)=u_{i}\left(x_{i}^{\varepsilon}\right) \delta_{i} \mu(S) \\
& \quad-u_{i}\left(x_{i}\right) \delta_{i} \mu(S \cup\{k\})-u_{k}\left(x_{k}\right) \delta_{i, k} \mu(S),
\end{aligned}
$$

and if $u_{i}\left(x_{i}^{\varepsilon}\right) \leq u_{k}\left(x_{k}\right)$

$U\left(x_{i}^{\varepsilon}, x_{-i}\right)-U(x)=\left(u_{i}\left(x_{i}^{\varepsilon}\right)-u_{i}\left(x_{i}\right)\right) \delta_{i} \mu(S \cup\{k\})$

where $S:=A_{>i}(x) \backslash\{k\}=\left\{j \in N \backslash\{i, k\}: u_{j}\left(x_{j}\right)>\right.$ $\left.u_{i}\left(x_{i}\right)\right\}$.

The situation proved in Lemma 1 is summarized in Figure 1.

In practice, we fix $x_{-k}$ and $\varepsilon>0$, and we study the shape of the function $x_{k} \mapsto U\left(x_{i}^{\varepsilon}, x_{-i}\right)-U(x)$. The shape can be either a constant function, or as described in Figure 1. In the first case, it is easy to find a necessary and sufficient condition, according to Lemma 1:

$$
\begin{aligned}
& \forall x_{k}, y_{k} \in X_{k} \\
& U(x)-U\left(x_{i}^{\prime}, x_{-i}\right)=U\left(y_{k}, x_{-k}\right)-U\left(x_{i}^{\prime}, y_{k}, x_{-i, k}\right) \\
& \Longleftrightarrow \delta_{i, k} \mu(S)=0 .
\end{aligned}
$$




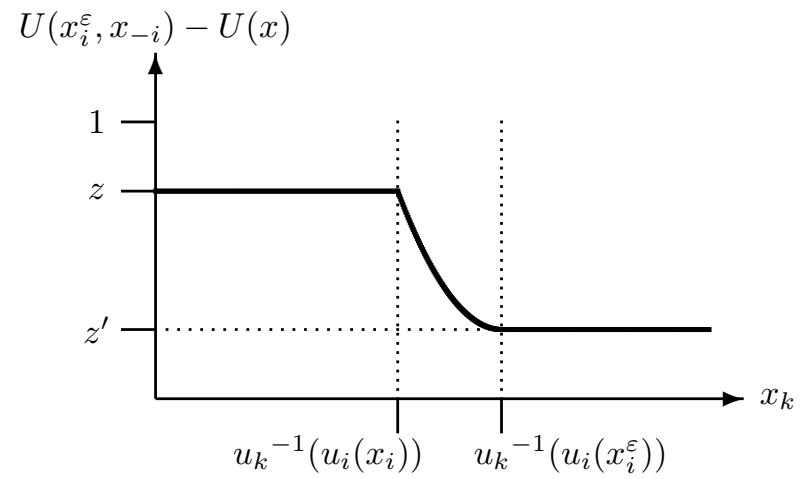

Figure 1: Function $x_{k} \mapsto U\left(x_{i}^{\varepsilon}, x_{-i}\right)-U(x)$ with $z:=\left(u_{i}\left(x_{i}^{\varepsilon}\right)-u_{i}\left(x_{i}\right)\right)[\mu(S \cup\{i\})-\mu(S)]$ and $z^{\prime}:=$ $\left(u_{i}\left(x_{i}^{\varepsilon}\right)-u_{i}\left(x_{i}\right)\right)[\mu(S \cup\{i, k\})-\mu(S \cup\{k\})]$.

The condition on $\varepsilon$ that is expressed in Lemma 1 is difficult to satisfy. Indeed, as the value functions are not a priori known (the goal is construct them), one cannot know whether $\varepsilon$ is sufficiently small so that so that $\left(u_{j}\left(x_{j}\right)\right)_{j \in N \backslash\{k\}}$ and $\left(u_{i}\left(x_{i}^{\varepsilon}\right),\left(u_{j}\left(x_{j}\right)\right)_{j \in N \backslash\{i, k\}}\right)$ are comonotone. The following lemma generalizes Lemma 1 in order to get rid of the assumption on $\varepsilon$.

Lemma 2 Let $x \in X$, and $\varepsilon>0$. We assume that there exists $p$ criteria $l_{1}, \ldots, l_{p}$ such that

$u_{i}\left(x_{i}\right)<u_{l_{1}}\left(x_{l_{1}}\right) \leq u_{l_{2}}\left(x_{l_{2}}\right) \leq \cdots \leq u_{l_{p}}\left(x_{l_{p}}\right)<u_{i}\left(x_{i}^{\varepsilon}\right)$.

Then if $u_{k}\left(x_{k}\right) \leq u_{i}\left(x_{i}\right)$

$$
\begin{aligned}
& U\left(x_{i}^{\varepsilon}, x_{-i}\right)-U(x) \\
& \left.=u_{i}\left(x_{i}^{\varepsilon}\right)\right) \delta_{i} \mu(S)-u_{i}\left(x_{i}\right) \delta_{i} \mu\left(S \cup\left\{l_{1}, \ldots, l_{p}\right\}\right) \\
& \quad-\sum_{m=1}^{p} u_{l_{m}}\left(x_{l_{m}}\right) \delta_{i, l_{m}} \mu\left(S \cup\left\{l_{m+1}, \ldots, l_{p}\right\}\right),
\end{aligned}
$$

and if $u_{i}\left(x_{i}^{\varepsilon}\right) \leq u_{k}\left(x_{k}\right)$

$$
\begin{aligned}
& U\left(x_{i}^{\varepsilon}, x_{-i}\right)-U(x)= \\
& \left.=u_{i}\left(x_{i}^{\varepsilon}\right)\right) \delta_{i} \mu(S \cup\{k\}) \\
& \quad-u_{i}\left(x_{i}\right) \delta_{i} \mu\left(S \cup\left\{l_{1}, \ldots, l_{p}, k\right\}\right) \\
& \quad-\sum_{m=1}^{p} u_{l_{m}}\left(x_{l_{m}}\right) \delta_{i, l_{m}} \mu\left(S \cup\left\{l_{m+1}, \ldots, l_{p}, k\right\}\right),
\end{aligned}
$$

where $S:=\left\{j \in N \backslash\{i, k\}: u_{j}\left(x_{j}\right) \geq u_{i}\left(x_{i}^{\varepsilon}\right)\right\}$.

We have the following result.

Theorem 1 Let $x_{i} \in X_{i}$ and $\varepsilon>0$. Then one of the following two cases holds:

(i) Either there exists $x_{-i, k} \in X_{-i, k}$ such that the function $x_{k} \mapsto U\left(x_{i}^{\varepsilon}, x_{-i}\right)-U(x)$ is not constant. Then the largest value $x_{k}^{\star}$ of $x_{k}$ such that for all $x_{k}^{\prime} \leq x_{k}, U\left(x_{i}^{\varepsilon}, x_{-i}\right)-U(x)=$ $U\left(x_{i}^{\varepsilon}, x_{k}^{\prime}, x_{-i, k}\right)-\bar{U}\left(x_{k}^{\prime}, x_{-k}\right)$ satisfies to the relation

$$
u_{k}\left(x_{k}^{\star}\right)=u_{i}\left(x_{i}\right)
$$

(ii) Or for all $x_{-i, k} \in X_{-i, k}$, the function $x_{k} \mapsto$ $U\left(x_{i}^{\varepsilon}, x_{-i}\right)-U(x)$ is constant. Then

$$
\forall A \subseteq N \backslash\{i, k\} \quad \delta_{i, k} \mu(A)=0 .
$$

If we are in the situation (i) in Theorem 1, the element $x_{k}^{\star}$ that is constructed from $x_{i}$ is denoted by $\mathrm{Comm}_{i \rightarrow k}\left(x_{i}\right)$, where Comm stands for commensurateness. We are indeed able to find an element on attribute $k$ that is commensurate with the element $x_{i}$ on attribute $i$.

Situation (ii) in Theorem 1 implies that criteria $i$ and $k$ are independent (see (9)). The condition that $x_{k} \mapsto U\left(x_{i}^{\varepsilon}, x_{-i}\right)-U(x)$ is constant can be expressed in terms of the quaternary relation $\succsim^{\star}$ :

$\forall x_{k}, x_{k}^{\prime} \in X_{k} \quad\left(x_{i}^{\varepsilon}, x_{-i}\right) x \sim^{\star}\left(x_{i}^{\varepsilon}, x_{k}^{\prime}, x_{-i, k}\right)\left(x_{k}^{\prime}, x_{-k}\right)$.

This latter condition expressed in the attribute space characterizes the absence of interaction between criteria $i$ and $k$. This is an important consequence of this theorem.

\subsection{Commensurateness between subsets of criteria}

We introduce the Algorithm 1 to construct a partition $\mathcal{T}$ of $N$ and a vector $x \in \mathbb{R}^{N}$. In the algorithm, $\operatorname{get} \operatorname{Value}(j)$ is a function that generates a random element of $X_{j}$.

Function getCommensurateSequence () :

$\mathcal{T} \leftarrow \emptyset$ and $R \leftarrow N$;

While $(R \neq \emptyset)$ do

Select $j \in R$

$x_{j} \leftarrow \operatorname{getValue}(j)$

$S \leftarrow\{j\}$ and $R \leftarrow R \backslash\{j\}$;

While $(\exists i \in S, k \in R$ such that situation (i) in Theorem 1 holds) do

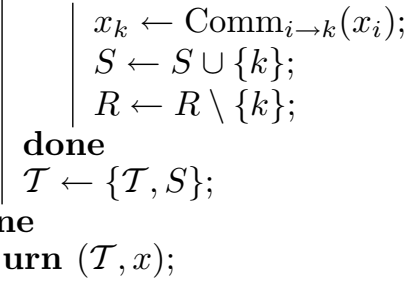

\section{End}

done

return $(\mathcal{T}, x)$

Algorithm 1: Algorithm for the determination of $\mathcal{T}$ and the vector $x$.

Theorem 2 Let $(\mathcal{T}, x)$ be the outcome of the function "getCommensurateSequence" (see Algorithm 1), where getValue $(j)$ is a function that generates a random element of $X_{j}$. Then $\mathcal{T}$ forms a partition of $N$.

For all $S \in \mathcal{T}$, we have

$$
\forall i, k \in S \quad u_{i}\left(x_{i}\right)=u_{k}\left(x_{k}\right) .
$$


Moreover,

$$
\forall A \subseteq N \quad \mu(A)=\sum_{S \in \mathcal{T}} \mu(A \cap S) .
$$

The previous theorem proves that the set of criteria can be partitioned into disjoint sets such that all elements of $x$ in each coalition are commensurate (they have the same value), and each set is independent of the other ones (see Figure 2).

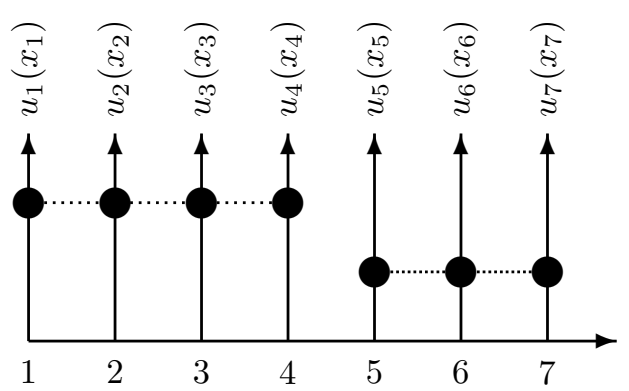

Figure 2: Example $\mathcal{T}=\{\{1,2,3,4\},\{5,6,7\}\}$. We have $u_{1}\left(x_{1}\right)=u_{2}\left(x_{2}\right)=u_{3}\left(x_{3}\right)=u_{4}\left(x_{4}\right)$ and $u_{5}\left(x_{5}\right)=u_{6}\left(x_{6}\right)=u_{7}\left(x_{7}\right)$.

Lemma 3 Let $\mathcal{T}$ be a partition of $N$. Assume that for all $S, T \in \mathcal{T}$ Assume that

$$
\forall i \in S \forall j \in T \forall A \subseteq N \backslash\{i, j\} \quad \delta_{i, j} \mu(A)=0 .
$$

Then

$$
\forall A \subseteq N \quad \mu(A)=\sum_{S \in \mathcal{T}} \mu(A \cap S)
$$

The previous lemma shows that the different coalitions in $\mathcal{T}$ are independent.

\section{Construction of the aggregation function and value functions}

\subsection{Construction of the value functions on the positive part of the scales}

Following [7], it is possible to construct the value functions on a bounded unipolar scale through a Choquet integral with an unknown capacity as soon as one can identify on each attribute two reference levels $\mathbb{O}$ and $\mathbb{G}(\mathbb{G}$ stands for $G o o d)$ on each attribute. For unbounded bipolar scales, one needs three reference levels $\mathbb{B}$ (B stands for $B a d), \mathbb{O}$ and $\mathbb{G}$ to construct a value function [25], with the property that $u_{i}\left(\mathbb{G}_{i}\right)=1=-u_{i}\left(\mathbb{B}_{i}\right)$. As the $\mathbb{B}$ and $\mathbb{G}$ levels are generated more or less randomly in our approach (see Section 4), it is impossible to ensure the previous property. Hence we can only construct the positive part of the scale using our approach, i.e. the value function for the elements of $X_{i}$ above $\mathbb{O}_{i}$.

We first construct two reference levels $\mathbb{O}$ and $\mathbb{G}$. The idea is thus to use twice the process described in Section 4 to construct these two levels.
Definition 2 The outcome of function "getCommensurateSequence" in Algorithm 1 is denoted by $(\mathcal{T}, \mathbb{O})$ where function get Value $(j)$ generates any element in $X_{j}$.

Function "getCommensurateSequence" in Algorithm 1 is used a second time and its outcome is denoted by $(\mathcal{T}, \mathbb{G})$ where function getValue $(j)$ generates any element in $X_{j}$ that is strictly preferred to $\mathbb{O}_{j}$.

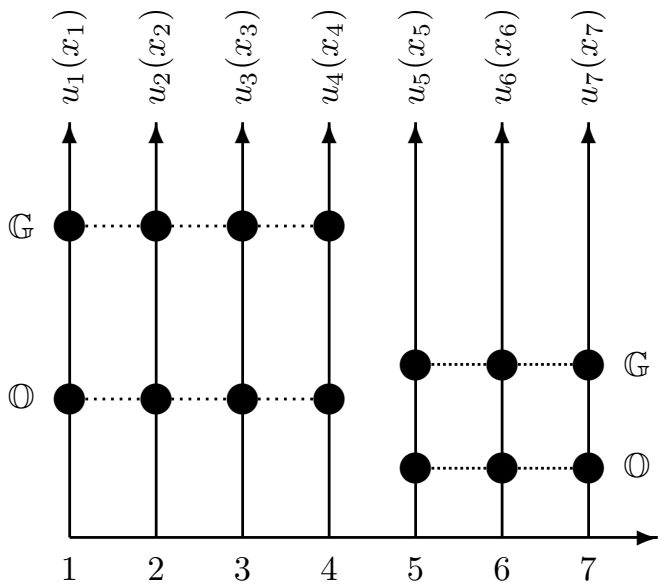

Figure 3: Example $\mathcal{T}=\{\{1,2,3,4\},\{5,6,7\}\}$.

It is apparent that Algorithm 1 always produces the same partition $\mathcal{T}$ whatever the values that are generated in get $\operatorname{Value}(j)$.

We define for all $x_{i} \in X_{i}$ with $x_{i} \geq \mathbb{O}_{i}$

$$
v_{i}\left(x_{i}\right)=\frac{U\left(x_{i}, \mathbb{O}_{-i}\right)-U\left(\mathbb{O}_{N}\right)}{U\left(\mathbb{G}_{i}, \mathbb{O}_{-i}\right)-U\left(\mathbb{O}_{N}\right)} .
$$

With this definition, we have the following normalization

$$
v_{i}\left(\mathbb{G}_{i}\right)=1 \quad \text { and } \quad v_{i}\left(\mathbb{O}_{i}\right)=0 .
$$

\subsection{Identification of the capacity}

For each $S \in \mathcal{T}$, we define a capacity $\nu_{S}$ on $S$ by

$$
\forall A \subseteq S \quad \nu_{S}(A)=\frac{U\left(\mathbb{G}_{A}, \mathbb{O}_{-A}\right)-U\left(\mathbb{O}_{N}\right)}{U\left(\mathbb{G}_{S}, \mathbb{O}_{-S}\right)-U\left(\mathbb{O}_{N}\right)}
$$

We set $\nu=\left\{\nu_{S}\right\}_{S \in \mathcal{T}}$.

\subsection{Construction of the value functions on the negative part of the scale}

The value function on the negative part is obtained as follows. We define for all $x_{i} \in X_{i}$ with $x_{i} \leq \mathbb{O}_{i}$

$$
v_{i}\left(x_{i}\right)=\frac{U\left(x_{i}, \mathbb{O}_{-i}\right)-U\left(\mathbb{O}_{N}\right)}{U\left(\mathbb{G}_{N}\right)-U\left(\mathbb{O}_{i}, \mathbb{G}_{-i}\right)} .
$$

\subsection{Main result}

Theorem 3 Let us consider an overall value function $U$ that takes the form (5), where $u_{1}, \ldots, u_{n}, \mu$ are unknown. 
Let $\mathcal{T}, \mathbb{O}, \mathbb{G}$ defined by Definition $2, v_{i}$ defined by (12) and (14), and $\nu$ defined by (13). Then for all $x \in X$

$$
\begin{aligned}
& U(x)=U\left(\mathbb{O}_{N}\right) \\
& \quad+\sum_{S \in \mathcal{T}}\left(U\left(\mathbb{G}_{S}, \mathbb{O}_{-S}\right)-U\left(\mathbb{O}_{N}\right)\right) C_{\nu_{S}}\left(v_{S}\left(x_{S}\right)\right)
\end{aligned}
$$

where $v_{S}\left(x_{S}\right)=\left(v_{i_{1}}\left(x_{i_{1}}\right), v_{i_{2}}\left(x_{i_{2}}\right), \ldots, v_{i_{s}}\left(x_{i_{s}}\right)\right.$ if $S$ writes $S=\left\{i_{1}, i_{2}, \ldots, i_{s}\right\}$.

$$
\text { Let us consider two construc- }
$$

tions $\left\langle\mathcal{T}, \mathbb{O}, \mathbb{G}, v_{1}, \ldots, v_{n}, \nu\right\rangle \quad$ and $\left\langle\mathcal{T}^{\prime}, \mathbb{O}^{\prime}, \mathbb{G}^{\prime}, v_{1}^{\prime}, \ldots, v_{n}^{\prime}, \nu^{\prime}\right\rangle$ from $U$. Then $\mathcal{T}^{\prime}=\mathcal{T}$ and $\nu^{\prime}=\nu$. Moreover, for all $S \in \mathcal{T}$ there exists $\alpha_{S}>0$ and $\beta_{S} \in \mathbb{R}$ such that

$$
\forall i \in S \quad \forall x_{i} \in X_{i} \quad v_{i}^{\prime}\left(x_{i}\right)=\alpha_{S} v_{i}\left(x_{i}\right)+\beta_{S} .
$$

This result shows that all compatible scales are identical up to an affine transformation. The renormalization are different in each set of the partition $\mathcal{T}$. Moreover, the capacities defined on each set of the partition are uniquely defined.

Lemma 4 Let $\mathcal{T}$ be a partition of $N$. Assume that

$$
\forall A \subseteq N \quad \mu(A)=\sum_{S \in \mathcal{T}} \mu(A \cap S) .
$$

Then for all $a \in \mathbb{R}^{N}$

$$
C_{\mu}(a)=\sum_{S \in \mathcal{T}} C_{\mu}\left(a_{S}, 0_{-S}\right) .
$$

From the previous lemma, the Choquet integral is just the sum of the aggregation functions over each $S \in \mathcal{T}$. This is the form of the GAI network that generalizes the additive value model [26].

Corollary 1 We consider a pair of commensurate profiles $\mathbb{O}, \mathbb{G}$ such that

$$
\sum_{S \in \mathcal{T}}\left(U\left(\mathbb{G}_{S}, \mathbb{O}_{-S}\right)-U\left(\mathbb{O}_{N}\right)\right)=1
$$

It is always possible to construct profiles satisfying this relation.

Under the condition of Theorem 3, let us define a set function $\widehat{\mu}$ over $N$ by

$$
\begin{aligned}
& \forall S \in \mathcal{T} \forall A \subseteq S \\
& \quad \widehat{\mu}(A)=\left(U\left(\mathbb{G}_{S}, \mathbb{O}_{-S}\right)-U\left(\mathbb{O}_{N}\right)\right) \nu_{S}(A), \\
& \forall A \subseteq N \quad \widehat{\mu}(A)=\sum_{S \in \mathcal{T}} \widehat{\mu}(A \cap S),
\end{aligned}
$$

and we define $\widehat{v}_{i}$ by

$$
\widehat{v}_{i}\left(x_{i}\right)=v_{i}\left(x_{i}\right)+U\left(\mathbb{O}_{N}\right) .
$$

Then $\widehat{\mu}$ is a capacity, and for all $x \in X$

$$
U(x)=C_{\widehat{\mu}}\left(\widehat{v}_{1}\left(x_{1}\right), \ldots, \widehat{v}_{n}\left(x_{n}\right)\right) .
$$

Let us consider two constructions $\left\langle\mathcal{T}, \mathbb{O}, \mathbb{G}, \widehat{v}_{1}, \ldots, \widehat{v}_{n}, \widehat{\mu}\right\rangle$ and $\left\langle\mathcal{T}, \mathbb{O}^{\prime}, \mathbb{G}^{\prime}, \widehat{v}_{1}^{\prime}, \ldots, \widehat{v}_{n}^{\prime}, \widehat{\mu}^{\prime}\right\rangle$ satisfying (16). Then for all $S \in \mathcal{T}$ there exists $\alpha_{S}>0$ and $\beta_{S} \in \mathbb{R}$ such that

$$
\begin{aligned}
& \forall i \in S \quad \forall x_{i} \in X_{i} \quad \widehat{v}_{i}^{\prime}\left(x_{i}\right)=\alpha_{S} \widehat{v}_{i}\left(x_{i}\right)+\beta_{S}, \\
& \widehat{\mu}^{\prime}(S)=\frac{\widehat{\mu}(S)}{\alpha_{S}} .
\end{aligned}
$$

Condition (16) is the normalization condition. It implies that the game $\widehat{\mu}$ that is constructed, is a capacity.

In the particular case where all criteria are independent, which corresponds to the weighted sum model, it is not possible to construct a sequence of commensurate elements of the attributes. One can merely take any vector of $X$ for $\mathbb{O}$ and $\mathbb{G}$. Hence the unit $\widehat{v}_{i}\left(\mathbb{G}_{i}\right)-\widehat{v}_{i}\left(\mathbb{O}_{i}\right)$ has no intrinsic meaning in this case. From Corollary 1, we have

$$
U(x)=\sum_{i \in N}\left(U\left(\mathbb{G}_{i}, \mathbb{O}_{-i}\right)-U\left(\mathbb{O}_{N}\right)\right) \widehat{v}_{i}(x) .
$$

This means that the factor $\left.U\left(\mathbb{G}_{i}, \mathbb{O}_{-i}\right)-U\left(\mathbb{O}_{N}\right)\right)$, which is usually considered as representing the importance of criterion $i$, appears here as being only a normalization factor. The condition (16) is the normalization condition. We are thus closer to the additive value model than to a true weighted sum model in which the scales are really commensurate.

Concerning the second part of the previous corollary, it says that if the scale $\widehat{v}$ is transformed by an affine transformation in a coalition $S \in \mathcal{T}$, then the capacity $\widehat{\mu}$ of $S$ shall be transformed by the inverse of the affine transformation. As a whole the overall utility thus remains the same.

This cannot be done when the partition is composed of only one set. Hence the following result is clear.

Corollary 2 The construction is unique if and only if the partition $\mathcal{T}$ that is constructed is composed on only the grand coalition $N$.

\section{Conclusion}

One of the main difficulty in the application of the Choquet integral in multi-criteria decision making is the necessity to make the value function commensurate. The former approaches in the literature always assume commensurateness. But this hypothesis is often very hard to obtain in practice.

This paper proposes an approach to construct the Choquet integral without any prior commensurateness assumption. The idea is that if the value of criterion $\mathrm{k}$ varies and the other criteria are fixed, then one may observe that the weight of criterion $\mathrm{i}$ suddenly changes when the value of criterion $\mathrm{k}$ is equal to that of criterion i. This enables us to 
construct sequences of commensurate values on the different attributes.

For the sake of simplicity, we assumed here that the value functions are strictly monotone. The case of strictly decreasing values would be managed in the same way. It is apparent that one could also manage the case of a non-monotone value function. One could indeed discover the type of (piecewise) monotonicity of a value function by changing only the value on this attribute and analyzing the variation of the overall utility.

\section{References}

[1] J. Von Neuman and O. Morgenstern. Theory of Games and Economic Behavior. Princeton University Press, 1944.

[2] L. J. Savage. The Foundations of Statistics. Dover, 2nd edition, 1972.

[3] D.H. Krantz, R.D. Luce, P. Suppes, and A. Tversky. Foundations of measurement, volume 1: Additive and Polynomial Representations. Academic Press, 1971.

[4] D. Schmeidler. Subjective probability and expected utility without additivity. Econometrica, 57(3):571-587, 1989.

[5] M. Grabisch. The application of fuzzy integrals in multicriteria decision making. European $\mathrm{J}$. of Operational Research, 89:445-456, 1996.

[6] S. Angilella, S. Greco, F. Lamantia, and B. Matarazzo. Assessing non-additive utility for multicriteria decision aid. European Journal of Operational Research, 158:734-744, 2004.

[7] Ch. Labreuche and M. Grabisch. The Choquet integral for the aggregation of interval scales in multicriteria decision making. Fuzzy Sets 89 Systems, 137:11-26, 2003.

[8] J.-L. Marichal. An axiomatic approach of the discrete Choquet integral as a tool to aggregate interacting criteria. IEEE Tr. on Fuzzy Systems, 8(6):800-807, 2000.

[9] M. Couceiro and J.L. Marichal. Axiomatizations of quasi-lovàsz extensions of pseudiboolean functions. Preprint.

[10] Ch. Labreuche and M. Grabisch. The representation of conditional relative importance between criteria. Annals of Operation Research, 154:93-122, 2007.

[11] R. Slowinsli, S. Greco, and B. matarazzo. Axiomatization of utility, outranking and decision-rule preference models for multicriteria classification. Control and Cybernetics, 31(4):1005-1035, 2002.

[12] S. Greco, B. Matarazzo, and R. Słowinski. The axiomatic basis of Sugeno integral and associative aggregation operator. In 4th Int. Workshop on Preferences and Decisions, pages 3338, Trento, Italy, September 2003.

[13] S. Greco, B. Matarazzo, and R. Słowinski. Axiomatic characterization of a general utility function in terms of conjoint measurement and rough-set decision rules. European Journal of Operational Research, 158:271-292, 2004.

[14] D. Bouyssou and T. Marchant. An axiomatic approach to noncompensatory sorting methods in mcdm i: The case of two categories. European Journal of Operational Research, 178(1):217-245, 2007.

[15] B. Peleg and P. Sudholter. Introduction to the theory of cooperative games. Kluwer academic publisher, Boston, 2003.

[16] M. Grabisch, T. Murofushi, and M. Sugeno. Fuzzy Measures and Integrals. Theory and Applications (edited volume). Studies in Fuzziness. Physica Verlag, 2000.

[17] G. Choquet. Theory of capacities. Annales de l'Institut Fourier, 5:131-295, 1953.

[18] L. S. Shapley. A value for $n$-person games. In H. W. Kuhn and A. W. Tucker, editors, Contributions to the Theory of Games, Vol. II, number 28 in Annals of Mathematics Studies, pages 307-317. Princeton University Press, 1953.

[19] T. Murofushi and S. Soneda. Techniques for reading fuzzy measures (III): interaction index. In 9th Fuzzy System Symposium, pages 693696, Sapporo, Japan, May 1993. In Japanese.

[20] J.S. Dyer and R.K. Sarin. Measurable multiattribute value functions. Operations Research, 27:810-822, 1979.

[21] R. L. Keeney and H. Raiffa. Decision with Multiple Objectives. Wiley, New York, 1976.

[22] C.A. Bana e Costa and J.C. Vansnick. A theoretical framework for Measuring Attractiveness by a Categorical Based Evaluation TecHnique (MACBETH). In Proc. XIth Int. Conf. on MultiCriteria Decision Making, pages 1524, Coimbra, Portugal, August 1994.

[23] T. L. Saaty. A scaling method for priorities in hierarchical structures. J. Math. Psychology, 15:234-281, 1977.

[24] M. Grabisch and Ch. Labreuche. A decade of application of the Choquet and Sugeno integrals in multi-criteria decision aid. Quarterly Journal of Operations Research (4OR), 6:1-44, 2008.

[25] Ch. Labreuche and M. Grabisch. Generalized Choquet-like aggregation functions for handling ratio scales. European Journal of Operational Research, 172:931-955, 2006.

[26] Ch. Gonzales and P. Perny. Gai networks for utility elicitation. In Proceedings of the 9th International Conference on the Principles of Knowledge Representation and Reasoning, pages 224-234, 2004. 